EPJ Web of Conferences 36, 00002 (2012)

DOI: $10.1051 /$ epjconf/20123600002

(C) Owned by the authors, published by EDP Sciences, 2012

\title{
Sensitivity of the final resonance spectra on the hydrodynamical freeze out
}

\author{
J. Steinheimer ${ }^{1, a}$, J. Aichelin², and M. Bleicher ${ }^{3}$ \\ 1 Lawrence Berkeley National Laboratory, 1 Cyclotron Road, Berkeley, CA 94720, USA \\ 2 Subatech (UMR 6457, IN2P3/CNRS), Université de Nantes, Ecole des Mines de Nantes, 4 rue \\ Alfred Kastler, 44307 Nantes cedex 3, France \\ 3 Frankfurt Institute for Advanced Studies, Johann Wolfgang Goethe \\ Universität, Ruth-Moufang-Str. 1, 60438 Frankfurt am Main, Germany
}

\begin{abstract}
We present results on hadronic resonance production in high energy nuclear collisions from the UrQMD hybrid model. In particular we are interested in the effect of the final hadronic stage on the properties of resonances observable at RHIC and LHC experiments. We investigate weather these observable properties can be used to pinpoint the transition energy density from the QGP phase to the hadronic phase.
\end{abstract}

\section{Introduction}

The interpretation of the results of ultrarelativistic heavy ion collisions is one of the most challenging problems in theoretical nuclear physics presently. In these collisions, presently investigated at the Relativistic Heavy Ion Collider (RHIC) at the Brookhaven national laboratory and at the Large Hadron Collider (LHC) at CERN, more than thousand particles are observed in central collisions. Although the multiplicity and the single particle transverse momentum spectra at midrapidity of the different particle species are of interest by their own right, the purpose of the experiments is to find out whether during the reaction the matter has made a transition towards a new state of matter, a Quark-Gluon Plasma (QGP). This information is not directly visible in the measured spectra and therefore theoretical approaches have to be employed to figure out whether the measured observables are compatible with the existence of such a QGP or, even more wanted, whether they can even lead to the conclusion that such a state is necessary to explain the measured quantities.

It turned out that the multiplicity of the almost all different hadron species is compatible with the assumption that they are emitted from an equilibrated source characterized by a temperature $T$ and a chemical potential $\mu_{B}[1-3]$. The temperature which describes the data is very close to $T_{c}$, the critical temperature at which, according to lattice gauge calculations $[4,5]$, the transition between the QGP and the hadrons takes place. If a system reaches thermal equilibrium all knowledge about the history of the system is lost. Therefore the efforts to learn something about the time evolution of the system concentrates on those particles which do not agree with thermal model predictions. In particular interesting are protons at LHC and resonances at RHIC and LHC. It has been suggested that the lack of protons, and anti-protons, at the LHC is due to hadronic annihilation processes which takes place at the end of the expansion [6]. Here we concentrate on resonances.

If after hadronization the particles are still interacting one expects that the observed resonances are suppressed as compared to thermal model predictions. They are reconstructed by calculating the invariant mass of their decay products. If one of these decay products scatters on the way to the detector the resonance cannot be identified anymore. Due to their large mass we do not expect that the

\footnotetext{
a e-mail: jsfroschauer@lbl.gov
} 
resonances are regenerated at the end of the expansion phase because the average sqrts in collisions decreases constantly. This simple idea has been confirmed in UrQMD calculations [7].

In the meantime transport theory has been further developed and one has realized that the QGP at the beginning of the expansion is best described by a hydrodynamical approach. The equation of state which is used in these calculations and which has been calculated in lattice gauge calculations can be understood as a superposition of that of a QPG and that of a hadron resonance gas. At high density the contribution of the hadron resonance gas is almost negligible whereas close to the critical temperature the hadron resonance gas dominates. This allows to describe the transition from the hydrodynamical state to the hadron gas by the Cooper Frye formula. After hadronization the hadrons can still have interactions which may be the origin of the apparent suppression of resonances.

In this scenario it is the essential question whether the observed resonance yield reflects the energy density at which the transition between the hydrodynamical and the kinetic stage takes place. In these proceedings we will demonstrate that according to the results of the URQMD hybrid models indeed the finally observed spectra are close related to the critical energy density at which the transition takes place.

\section{The UrQMD Hybrid Model}

To investigate the effect of the hadronic final state on hadron resonances created at the hadronization, we employ the UrQMD hybrid model. The UrQMD hybrid model combines the advantages of a hadronic transport model with an intermediate hydrodynamical stage for the hot and dense phase of a heavy ion collision. The UrQMD Model $[8,9]$ (in its cascade mode) is used to calculate the initial state of a heavy ion collision for the hydrodynamical evolution [10]. This is done to account for the non-equilibrium dynamics in the very early stage of the collision. The coupling between the UrQMD initial state and the hydrodynamical evolution happens at a time $t_{\text {start }}$ when the two Lorentz-contracted nuclei have passed through each other. At this starting time all initial collisions have happened. It is further the earliest time at which local thermodynamical equilibrium may be achieved. At this time the energy, baryon number and momenta of all particles within UrQMD are mapped onto the spatial grid of the hydrodynamic model by representing each hadron by a Gaussian of finite width $\sigma=1 \mathrm{fm}$. In this approach the effects of event-by-event fluctuations and stopping of energy and baryon number density in the initial state are naturally included.

The full $(3+1)$ dimensional ideal hydrodynamic evolution is performed using the SHASTA algorithm $[11,12]$. We solve the equations for the conservation of energy and momentum and for the conservation of the baryonic current The partial differential equations are solved on a three-dimensional spatial Eulerian grid with fixed position and size $\delta x=0.2 \mathrm{fm}$ in the computational frame. To close the set of equations an equation of state (EoS), the pressure as function of energy and baryon number density $p(e, n)$ needs to be specified. In the following we will use an EoS that corresponds to an hadron resonance gas. This is convenient as it includes the same degrees of freedom as the UrQMD model, which essentially allows us to study the effect of local equilibrium on the different observables. Previous investigations have shown that different equations of state only lead to insignificant differences in the results $[13,14]$.

To transfer all particles back into the UrQMD model, an approximate iso-eigentime transition is chosen (see [15] for details). To this end we apply the Cooper Frye prescription [16] to individual transverse slices, of thickness $\Delta z=0.2 \mathrm{fm}$, at a time-like transition hypersurface. The transition time for a given slice is determined by the time when the energy density $\varepsilon$ in every cell of this slice has dropped below a certain transition energy density. As a result we obtain a longitudinal iso-eigentime transition with an almost rapidity independent maximum switching temperature for beam energies above $\sqrt{s_{N N}} \approx 10 \mathrm{GeV}$. In a given slice the hydrodynamic fields are transformed to particle degrees of freedom via the Cooper-Frye equation on an isochronous time-like hypersurface in the computational frame.

The above transition procedure conserves baryon number, electric charge, the total net strangeness and the total energy on an event-by-event basis. After the particles are created they evolve according to 


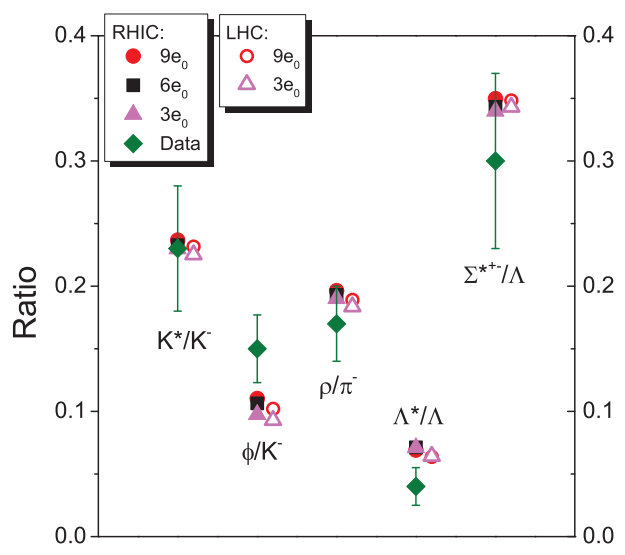

Fig. 1. Ratio of observed resonance yield to stable hadron. We show midrapidity $|y|<0.5$ ratios for most central collisions of $\mathrm{AuAu} / \mathrm{PbPb}$ at beam energies $\sqrt{s_{N N}}=200$ (full symbols) and $2700 \mathrm{GeV}$ (open symbols). The symbol styles depict different transition criteria for the hydrodynamical evolution. The green diamonds represent data at $\sqrt{S_{N N}}=200 \mathrm{GeV}[18]$.

a hadronic cascade (UrQMD) where final re-scatterings and decays are calculated until all interactions cease and the system decouples. A more detailed description of the hybrid model including parameter tests and results can be found in [17].

\section{Results}

In the following we present our results for the spectra and yields of resonances at the transition hypersurface and after the final hadronic state. In our calculation we identify an observable resonance by following the time evolution of its decay products. If all decay products do not rescatter we assume the resonance to be reconstructable from the final hadron measurements. On the other hand if a decay product of the resonance undergoes at least one rescattering we assume it to be not observable.

Figure 1 shows the final mid rapidity $(|y|<0.5)$ ratio of observable resonances to stable hadrons in simulations of $\mathrm{AuAu} / \mathrm{PbPb}$ collisions at $\sqrt{s_{N N}}=200$ and $2700 \mathrm{GeV}$. In particular we compare the results for different values of the transition energy density. We observe a very small energy dependence. Also the dependence of the final ratio on the transition criterion is rather small. In general our results follow the data with small deviations of the $\phi / K^{-}$ratio when compared to data.

To understand more of the dynamics of the resonances in the hadronic phase we investigate the time evolution of the decays and last scatterings of the different resonances created in AuAu collisions at $\sqrt{s_{N N}}=200 \mathrm{GeV}$. In the left part of figure 2 we show the decay rate of observable resonances, together with the rate of last interactions of these resonances, as a function of time. Here we observe that most resonances (except the $\rho$ ) decay a considerable time after their last scattering. The left hand side of figure 2 shows the time evolution of the gain (dashed) and loss (solid) rates of all resonances in the hadronic phase. Except for the $\phi$, which has a small hadronic cross section, all resonances have a considerable gain term for at least 10-20 fm after the transition into the hadronic phase.

The time evolution of the gain term, relative to the loss term, is shown in the left hand side of figure 3. All resonances show to have a considerable contribution from the final hadronic state. In particular the $\Lambda^{*}$ which has even a larger gain than loss term for a short period of time.

The fraction of observable resonances which has decayed until a point in time $t$ is shown on the right 

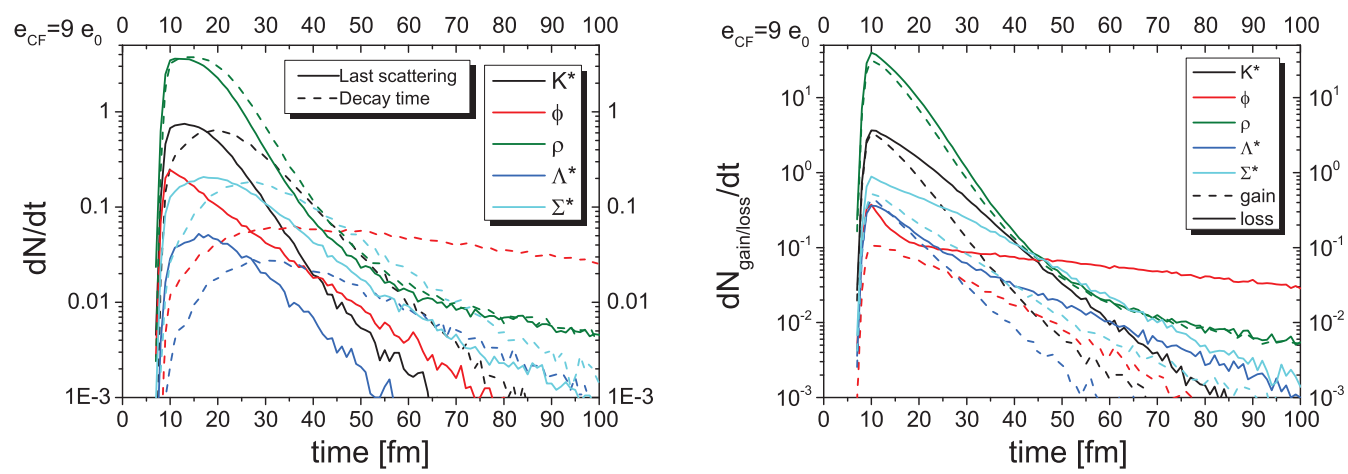

Fig. 2. Left: Number of observable resonances, decaying at a certain time $t$ as a function of time (dashed lines). The solid line depicts the number of observable resonances which undergo their last scattering at a time $t$ as a function of time. Right: Number of resonances produced (dashed line) and decaying (solid line) at a certain time $\mathrm{t}$, as a function of time. All results shown are for most central collisions of AuAu at $\sqrt{s_{N N}}=200 \mathrm{GeV}$ and with a transition criterion of $e_{C F}=9 e_{0}$.
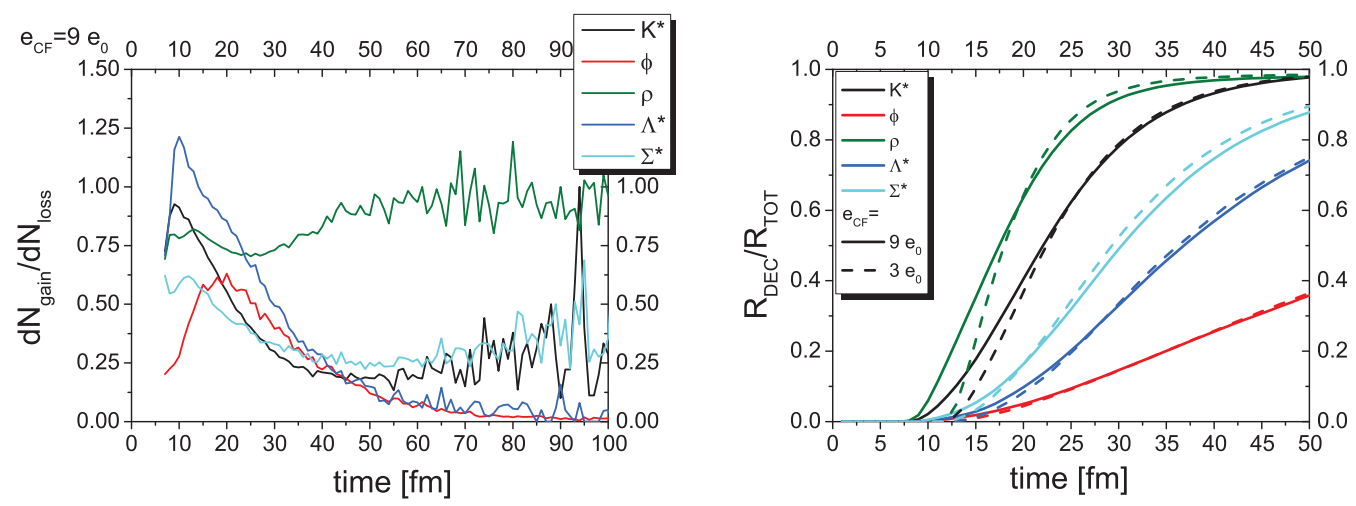

Fig. 3. Left: Ratio of resonances lost and created at a certain time, during the hadronic transport phase, as a function of time Right: Fraction of observable resonances which has decayed until a certain time. We compare results for two different transition criteria. (for a transition criterion of $e_{C F}=9 e_{0}$ ).

hand side of figure 2 . One can clearly see an ordering due to the different lifetimes of the resonances. While the $\rho$ 's have almost all decayed until $20 \mathrm{fm}$ after the transition, the $\phi$ lives considerably longer and decays at much later times.

Thus $\rho$ mesons are those which carry information about the early stage of the expansion because at $15 \mathrm{fm} / \mathrm{c} 50 \%$ of the finally observed $\rho$ have already been created. Nevertheless the many rescatterings during the hadronic phase wash out the information on the transition energy density. This explains why the total yield is that insensitive on the transition energy density, as seen in figure fig: 1 .

\section{$3.1 p_{\perp}$ Dependence}

In the following we will discuss the $p_{\perp}$ dependence of resonance modifications in the hadronic phase. For example we are interested to see a $p_{\perp}$ dependent absorption. This is displayed on the left hand side of figure 4 . We show the $p_{\perp}$ dependent ratio of observed resonances to those produced at the transition hypersurface. We also compare two different transition criteria, $3 e_{0}$ (solid lines) and $9 e_{0}$ (dashed lines). We observe that during the expansion the resonances gain a lot of transverse momentum. Therefore at low $p_{\perp}$ the ratio is below one while the spectra are enhanced at high $p_{\perp}$. This is mainly due to the collective flow the resonances gain in the final stage. 

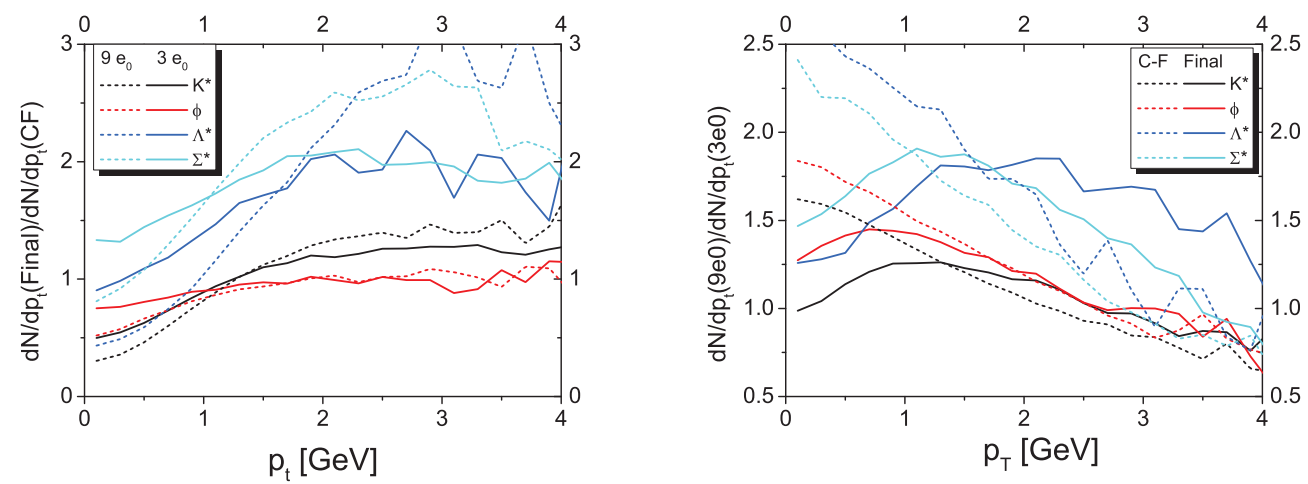

Fig. 4. Left: Ratio of the $p_{\perp}$ distribution of observable resonances to that of resonances produced at the CooperFrye transition, as a function of $p_{\perp}$. Right: Ratio of the $p_{\perp}$ distribution of resonances with a transition criterion of $e_{C F}=9 e_{0}$ to $e_{C F}=3 e_{0}$. The dashed line shows the ratio at the transition while the solid line is for observable resonances only. All results shown are for most central collisions of AuAu at $\sqrt{s_{N N}}=200 \mathrm{GeV}$ and with a transition criterion of $e_{C F}=9 e_{0}$ (dashed lines) or $3 e_{0}$ (solid lines).

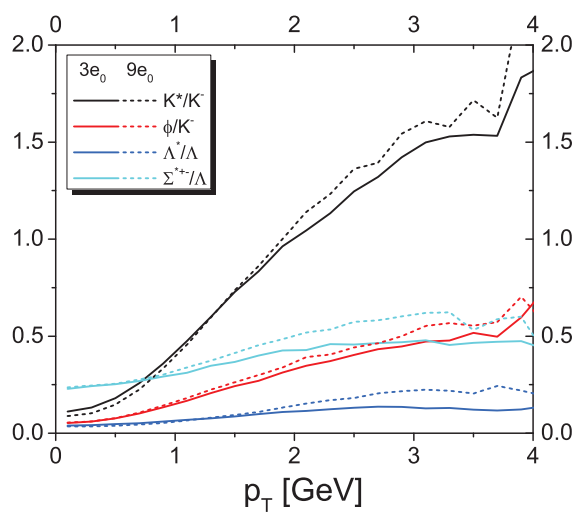

Fig. 5. Ratio of observable resonances to stable hadrons as a function of $p_{\perp}$. The lines are results for a transition criterion of $e_{C F}=3 e_{0}$ while the dashed lines depict results for $e_{C F}=9 e_{0}$.

In the right part of figure 4 we compare two different transition criteria as a function of $p_{\perp}$. The dashed lines depict results directly at the transition hypersurface, while the solid lines are for observable resonances. It is clear that the $p_{\perp}$ distributions for different transition densities differ considerably at the freeze out surface because a higher freeze out energy density correspond to a higher temperature. It is, however, astonishing that also the spectra of the finally observed resonances differ by up to a factor of 1.7 for the two freeze out energy densities. The figure show as well that the momentum shift or the collective flow depends strongly on the freeze out energy density.

Finally it is useful how the phenomena described above show up in observables. For this purpose we calculate the $p_{\perp}$ dependence of the ratios of observable resonances to stable hadrons. Figure 5 depicts these ratios for several resonances for two different transition criteria $3 e_{0}$ (solid lines) and $9 e_{0}$ (dashed lines). The ratios are very similar because the stable particles obtain a similar collective flow as the resonances. Only at large transverse momenta a difference can be observed. For the kaons this ratio may become as large as a factor of two. 


\section{Summary}

We have studied in this contribution whether resonances can be used to determine the transition energy density where hadronization occurs. We found that the collective flow in the hadronic phase depends strongly on this transition energy density. This collective flow affects, however, the stable particles and therefore the ratio of resonances to stable particles as a function of the transverse momentum shows only little dependence on the freeze out energy density. Whether the slope of the spectra can be used to determine the freeze out energy density remains to be seen.

\section{Acknowledgments}

This work was supported by BMBF, HGS-hire and the Hessian LOEWE initiative through the Helmholtz International center for FAIR (HIC for FAIR). J. S. acknowledges a Feodor Lynen fellowship of the Alexander von Humboldt foundation. This work was supported by the Office of Nuclear Physics in the US Department of Energy's Office of Science under Contract No. DE-AC02-05CH11231. The computational resources were provided by the LOEWE Frankfurt Center for Scientific Computing (LOEWE-CSC).

\section{References}

1. F. Becattini, M. Gazdzicki, A. Keranen, J. Manninen and R. Stock, Phys. Rev. C 69, 024905 (2004)

2. A. Andronic, P. Braun-Munzinger and J. Stachel, Phys. Lett. B 673, 142 (2009) [Erratum-ibid. B 678, $516(2009)]$

3. J. Cleymans, H. Oeschler, K. Redlich and S. Wheaton, Phys. Rev. C 73, 034905 (2006)

4. Y. Aoki, S. Borsanyi, S. Durr, Z. Fodor, S. D. Katz, S. Krieg and K. K. Szabo, JHEP 0906, 088 (2009)

5. A. Bazavov et al., Phys. Rev. D 85, 054503 (2012)

6. J. Steinheimer, J. Aichelin and M. Bleicher, arXiv:1203.5302 [nucl-th].

7. M. Bleicher and J. Aichelin, Phys. Lett. B 530, 81 (2002)

8. S. A. Bass et al., Prog. Part. Nucl. Phys. 41, 255 (1998) [Prog. Part. Nucl. Phys. 41, 225 (1998)]

9. M. Bleicher et al., J. Phys. G 25, 1859 (1999)

10. J. Steinheimer, M. Bleicher, H. Petersen, S. Schramm, H. Stocker and D. Zschiesche, Phys. Rev. C 77, $034901(2008)$

11. D. H. Rischke, S. Bernard and J. A. Maruhn, Nucl. Phys. A 595, 346 (1995)

12. D. H. Rischke, Y. Pursun and J. A. Maruhn, Nucl. Phys. A 595, 383 (1995) [Erratum-ibid. A 596, $717(1996)]$

13. H. Petersen, J. Steinheimer, M. Bleicher and H. Stocker, J. Phys. G 36, 055104 (2009)

14. J. Steinheimer, V. Dexheimer, H. Petersen, M. Bleicher, S. Schramm and H. Stoecker, Phys. Rev. C 81, 044913 (2010)

15. Q. f. Li, J. Steinheimer, H. Petersen, M. Bleicher and H. Stocker, Phys. Lett. B 674, 111 (2009)

16. F. Cooper and G. Frye, Phys. Rev. D 10, 186 (1974).

17. H. Petersen, J. Steinheimer, G. Burau, M. Bleicher and H. Stocker, Phys. Rev. C 78, 044901 (2008)

18. B. I. Abelev et al. [STAR Collaboration], Phys. Rev. C 78, 044906 (2008) 